\title{
Grounding, conceivability, and the mind-body problem
}

\author{
Hasen Khudairi ${ }^{1}$
}

Received: 11 January 2016 / Accepted: 22 October 2016 / Published online: 8 November 2016 (C) The Author(s) 2016. This article is published with open access at Springerlink.com

\begin{abstract}
This paper challenges the soundness of the two-dimensional conceivability argument against the derivation of phenomenal truths from physical truths (cf. Chalmers in The conscious mind, Oxford University Press, Oxford, 1996; The character of consciousness, Oxford University Press, Oxford, 2010) in light of a hyperintensional regimentation of the ontology of consciousness. The regimentation demonstrates how ontological dependencies between truths about consciousness and about physics cannot be witnessed by epistemic constraints, when the latter are recorded by the conceivability-i.e., the epistemic possibility-thereof. Generalizations and other aspects of the philosophical significance of the hyperintensional regimentation are further examined.
\end{abstract}

Keywords Consciousness · Grounding · Conceivability · Two-dimensional semantics

This paper argues that Chalmers's $(1996,2010)$ two-dimensional conceivability argument against the derivation of phenomenal truths from physical truths risks being obviated by a hyperintensional regimentation of the ontology of consciousness.

Chalmers (2010) provides the following argument against the identification of phenomenal truths with physical and functional truths. Let $\mathrm{M}$ be a model comprised of a domain D of formulas; C a set of epistemic possibilities; W a set of metaphysical possibilities; $\mathrm{R}_{c}$ and $\mathrm{R}_{w}$, accessibility relations on $\mathrm{C}$ and $\mathrm{W}$, respectively; and $\mathrm{V}$ a valuation function assigning formulas to subsets of $\mathrm{C}$ and W. So, $\mathrm{M}=\left\langle\mathrm{D}, \mathrm{C}, \mathrm{W}, \mathrm{R}_{c}, \mathrm{R}_{w}, \mathrm{~V}\right\rangle$. Let $\mathrm{P}$ denote the subset of formulas in the domain concerning fundamental physics, as well as both neurofunctional properties such as oscillations of neural populations,

\footnotetext{
$\bowtie$ Hasen Khudairi

hk44@st-andrews.ac.uk

1 Arché Philosophical Research Centre, University of St Andrews, 17-19 College Street, St Andrews, Fife KY16 9AL, Scotland
} 
and psychofunctional properties such as the retrieval of information from memory stores. Let Q denote the subset of formulas in the domain concerning phenomenal consciousness. A formula is epistemically necessary or apriori ( $\square$ ), if and only if it has the same value at all points in $\mathrm{C}$, if and only if it is impossible, i.e. inconceivable, for the formula to a variant value $(\neg \diamond \neg)$. A formula is negatively conceivable $(\diamond)$ if and only if nothing rules it out apriori ( $\neg \neg$ ) (144). A formula is metaphysically necessary if and only if it has the same value at all points in W. A formula is said to be 'super-rigid', if and only if it is both epistemically and metaphysically necessary, and thus has the same value at all points in epistemic and metaphysical modal space (2012, p. 474).

The physicalist thesis states that:

$\mathrm{P} \rightarrow \mathrm{Q}$.

Suppose, however, that the physicalist thesis is false. Thus,

1. $\neg(\mathrm{P} \rightarrow \mathrm{Q})$.

By the definition of the material conditional,

2. $\neg(\neg \mathrm{P} \vee \mathrm{Q})$.

By the De Morgan rules for negation,

3. $\neg \neg \mathrm{P} \wedge \neg \mathrm{Q}$.

By double negation elimination,

4. $\mathrm{P} \wedge \neg \mathrm{Q}^{1}$

The two-dimensional conceivability argument against physicalism proceeds as follows. ' $\mathrm{P} \wedge \neg \mathrm{Q}$ ' can receive a truth value relative to two parameters, a context, $\mathrm{C}$, and an index, W. In multi-dimensional intensional semantics, the value of the formula relative to the context determines the value of the formula relative to the index. Let the context range over a space of epistemic possibilities and let the index range over a space of metaphysical possibilities. Then,

$$
\llbracket \mathrm{P} \wedge \neg \mathrm{Q} \rrbracket^{c, w}=1 \text { iff } \exists \mathrm{c}^{\prime} \in \mathrm{C} \exists \mathrm{w}^{\prime} \in \mathrm{W} \llbracket \mathrm{P} \wedge \neg \mathrm{Q} \rrbracket^{c^{\prime}, w^{\prime}}=1 .^{2}
$$

The foregoing clause codifies the thought that, if it is epistemically possible that the truths about physics and functional organization obtain while the truths about consciousness do not, then the dissociation between $\mathrm{P}$ and Q is metaphysically possible as well. The argument depends on the assumption that propositions about consciousness and physics are super-rigid, such that the epistemic possibility concerning such truths can serve as a guide to the metaphysical possibility thereof.

If the conceivability argument is sound, then the physicalist thesis- that all phenomenal truths are derivable from physical and functional truths-is possibly false. The

\footnotetext{
1 For the formal equivalence, given the definition of the material conditional, see Chalmers (2010, p. 169).

${ }^{2}$ For the clause for the two-dimensional intension, see Chalmers and Rabern (2014, pp. 211-212). Chalmers' informal characterization of the argument proceeds as follows:

1. $\mathrm{P} \wedge \neg \mathrm{Q}$ is conceivable.

2. If $\mathrm{P} \wedge \neg \mathrm{Q}$ is conceivable, $\mathrm{P} \wedge \neg \mathrm{Q}$ is [epistemically, i.e.] 1-possible.

3. If $\mathrm{P} \wedge \neg \mathrm{Q}$ is 1-possible, $\mathrm{P} \wedge \neg \mathrm{Q}$ is [metaphysically, i.e.] 2-possible.

4. If $\mathrm{P} \wedge \neg \mathrm{Q}$ is 2-possible, then materialism is false. Thus,

5. Materialism is false (2010, p. 149).
} 
foregoing argument entrains, thereby, the metaphysical possibility of a property-based version of dualism between phenomenal consciousness and fundamental physics.

One of the standard responses to Chalmers's conceivability argument is to endeavor to argue that there are 'strong' necessities, i.e. cases according to which the necessity of the physical and phenomenal formulas throughout epistemic and metaphysical modal space is yet consistent with the epistemic possibility that the formulas have a different value. $^{3}$ Note, however, that strong necessities are ruled-out, just if one accepts the normal duality axioms for the modal operators: i.e., it is necessary that $\phi$ if and only if it is impossible for $\phi$ to be false: $\square \phi$ iff $\neg \diamond \neg \phi$. Thus, the epistemic necessity of $\phi$ rules out the epistemic possibility of not- $\phi$ by fiat. So, proponents of the strong necessity strategy are committed to a revision of the classical duality axioms.

Another line of counter-argument proceeds by suggesting that the formulas and terms at issue are not super-rigid. Against the super-rigidity of physical truths, one might argue, for example, that our knowledge of fundamental physics is incomplete, such that there might be newly discovered phenomenal or proto-phenomenal truths in physical theories from which the truths about consciousness might be derived. ${ }^{4}$ More contentiously, the epistemic profile of consciousness-as recorded by the concepts comprising our thoughts thereof, or by the appearance of its instantiation-might be dissociable from its actual instantiation. A variation on this reply takes our concepts of phenomenal consciousness still to refer to physical properties (cf. Block 2006). A related line of counter-argument relies on the assumption that phenomenal concepts are entities which are themselves physically reducible (cf. Balog 1999).

Finally, a counter-argument to the conceivability argument that has yet to be advanced in the literature is that its underlying logic might be non-classical. Thus, for example - by relying on double negation elimination in the inference from line 3 to 4 above - the equivalence between ' $\neg(\mathrm{P} \rightarrow \mathrm{Q})$ ' and ' $\mathrm{P} \wedge \neg \mathrm{Q}$ ' is intuitionistically invalid. A novel approach might further consist in arguing that epistemic modality might be governed by the Routley-Meyer semantics for relevant logic. ${ }^{5}$ Relevant validity can be defined via a ternary relation, such that $\llbracket \phi \rightarrow \psi \rrbracket^{\alpha}=1$ iff $\llbracket \phi \rrbracket^{\beta} \leq \llbracket \psi \rrbracket^{\gamma}$ and $\mathrm{R}(\alpha, \beta, \gamma)$, where the parameters, $\alpha, \beta$, and $\gamma$, range over epistemic possibilities. Then the irrelevant entailment, $\phi \wedge \neg \phi \rightarrow \psi$, can be avoided by setting $\llbracket \phi \rrbracket^{\beta}=1$; $\llbracket \phi \rrbracket^{\gamma}=0$; $\llbracket \psi \rrbracket^{\beta}=0$; while $\llbracket \psi \rrbracket^{\gamma}=1$. So, $\llbracket \phi \rrbracket^{\beta}=1$; $\llbracket \phi \rrbracket^{\gamma}=0$; and $\llbracket \psi \rrbracket^{\beta}=0$. The

\footnotetext{
3 As Chalmers (2010, pp. 166-167) writes, 'Before proceeding, it is useful to clarify [the general conceivability-possibility thesis] $\mathrm{CP}$ by making clear what a counterexample to it would involve ... Let us say that a negative strong necessity is a statement $\mathrm{S}$ such that $\mathrm{S}$ is [epistemically]-necessary and [metaphysically]-necessary but $\neg \mathrm{S}$ is negatively conceivable'. For a case-by-case examination of purported examples of strong necessities, see Chalmers (op. cit.: pp. 170-184, 2014). Because it is epistemically possible for there to be scenarios in which there is no consciousness, the target neighborhood of epistemically possible worlds is that in which the conditions on there being phenomenal consciousness are assumed to obtain. [Thanks here to Chalmers (p.c.).] Thus, the notion of epistemic necessity will satisfy conditions on real world validity, rather than general validity. In the latter case, a formula is necessary if and only if it has the same value in all worlds in a model. In the former case, the necessity at issue will hold throughout the neighborhood, where a neighborhood function assigns the subset of worlds in which consciousness obtains to a privileged world in the model.

4 See Seager (1995) and Strawson (2006) for the panpsychist proposal. Proponents of the pan-protopsychist approach include Stoljar (2001, 2014) and Montero (2010).

5 Cf. Routley (1972a,b) and Routley and Meyer (1973).
} 
philosophical interest of relevant logic is that it eschews the principle of disjunctive syllogism; i.e., $\forall \phi, \psi[[(\phi \vee \psi) \wedge \neg \phi] \rightarrow \psi]$ iff $\forall \phi, \psi[[\phi \wedge(\neg \phi \vee \psi)] \rightarrow \psi]$. Without disjunctive syllogism, logical entailment can no longer be identified with the material conditional, and this would block the derivation of line 2 from line 1 in the formal equivalence between ' $\neg(\mathrm{P} \rightarrow \mathrm{Q})$ ' and ' $\mathrm{P} \wedge \neg \mathrm{Q}$ '

In this essay, I will pursue a line of argument which is novel and distinct from the foregoing. I argue, in turn, that the conceivability argument can be circumvented, when the relationship between the truths about fundamental physics and the truths about phenomenal consciousness is analyzed in a classical, hyperintensional setting. Suppose, for example, that the physicalist thesis is defined using hyperintensional, grounding operators rather than metaphysical necessitation. ${ }^{6}$ Then, the epistemic and metaphysical possibility that $\neg(\mathrm{P} \rightarrow \mathrm{Q})$ is classically valid, although targets a less fine-grained metaphysical connection between physical and phenomenal truths. Even if P's grounding Q still entails the metaphysical necessitation of Q by $\mathrm{P}$, the epistemicintensional value of ' $\neg(\mathrm{P} \rightarrow \mathrm{Q})$ ' — will be an insufficient guide to the metaphysicalhyperintensional value of the proposition. So, even if the intension for 'consciousness' is rigid in both epistemic and metaphysical modal space, the epistemic intension recording the value of the proposition will be blind to its actual metaphysical value, because the latter will be hyperintensional.

In the remainder of this essay, I will outline the regimentation of the proposals in the ontology of consciousness using hyperintensional grounding operators, rather than the resources of modality and identity. ${ }^{7}$ By contrast to the modal approach underlying the conceivability argument, the hyperintensional regimentation targets the properties of reflexivity and bijective mappings, in order to countenance novel, ontological dependence relations between the properties of consciousness and physics, which are finer-grained than necessitation. ${ }^{8}$

Following Fine (2012a, b), let a polyadic operator have a ground-theoretic interpretation, only if the profile induced by the interpretation concerns the hyperintensional truth-making connection between an antecedent set of truths or properties and the relevant consequent. Let a grounding operator be weak if and only if it induces reflexive grounding; i.e., if and only if it is sufficient for the provision of its own ground. A grounding operator is strict if and only if it is not weak. A grounding operator is full if and only if it uniquely provides the explanatory ground for a fact. A grounding operator is part if and only if it - along with other facts-provide the explanatory ground for a fusion of facts.

Combinations of the foregoing explanatory operators may also obtain: $\mathrm{x}<\mathrm{y}$ iff $\phi$ is a strict full ground for $\psi ; \mathrm{x} \leq \mathrm{y}$ iff $\phi$ is a weak full ground for $\psi ; \mathrm{x} \prec \mathrm{y}$ iff $\phi$ is a strict part ground for $\psi ; \mathrm{x} \preceq \mathrm{y}$ iff $\phi$ is a weak part ground for $\psi ; \mathrm{x} \preceq \mathrm{y} \wedge \neg(\mathrm{y} \preceq \mathrm{x})$ iff $\phi$ is a strict partial ground for $\psi ; \mathrm{x} \prec * \mathrm{y}$ iff $\mathrm{x}_{1}, \ldots, \mathrm{x}_{n} \leq \mathrm{y}$, iff $\phi$ is a partial strict

\footnotetext{
${ }^{6}$ For the logic and operator-based semantics for the notion of explanatory ground, see Fine (2012a,b).

7 Cf. Khudairi (submitted), for the regimentation and for further discussion.

8 The claim that necessitation must be present in cases in which there is grounding is open to counterexample. Because, e.g., hyperintensional dependencies can obtain in only parts of, rather than entirely within, a world, the hyperintensional dependencies need not reflect necessitation. For further discussion of the grounding-necessitation thesis, see Rosen (2010) and Skiles (2015).
} 
ground for $\psi ; \mathrm{x} \prec^{\prime} \mathrm{z}$ iff $\left[\phi \prec^{*} \psi \wedge \psi \preceq \mu\right]$ iff $\phi$ is a part strict ground for some further fact, $\mu{ }^{9}$

The proposals in the metaphysics of consciousness can then be regimented in the hyperintensional framework as follows.

- Functionalism (modally: truths about consciousness are identical to truths about neuro- or psychofunctional role):

Functional truths (F) ground truths about consciousness (Q) if and only if the grounding operator is:

-strict full, s.t. F $<\mathrm{Q}$

-distributive (i.e. bijective between each truth-ground and grounded truth), s.t. $\exists f_{1-1}\langle\mathrm{~F}, \mathrm{Q}\rangle$

- Phenomenal Realist Type Identity (modally: truths about consciousness are identical to truths about biological properties, yet phenomenal properties are-in some sense-non-reductively real). ${ }^{10}$

Biological truths (B) ground truths about consciousness (Q) if and only if the grounding operator is:

-strict partial, s.t. $\mathrm{B} \preceq \mathrm{Q} \wedge \neg \mathrm{Q} \preceq \mathrm{B}$;

-distributive, s.t. $\exists f_{1-1}\langle\mathrm{~B}, \mathrm{Q}\rangle$; and

-truths about consciousness are weak part (i.e. the set partly reflexively grounds itself), s.t. $\mathrm{Q} \preceq \mathrm{Q}$

- Property Dualism (modally: truths about consciousness are identical neither to functional nor biological truths, yet are necessitated by physical truths):

Physical truths (P) ground truths about consciousness (Q) if and only if the grounding operator is:

-P $\preceq$ Q ;

-non-distributive, s.t. $\neg \exists f_{1-1}\langle\mathrm{P}, \mathrm{Q}\rangle$; and

9 The derivation is induced by the following proof-rules:

- Subsumption

$(<, \leq)$ :

$$
\left[\left(\mathrm{x}_{1}, \ldots, \mathrm{x}_{n}<\mathrm{y}\right)\right] \rightarrow(\mathrm{x} \leq \mathrm{y})
$$

$(<, \prec)$ :

$$
\left[\left(\mathrm{x}_{1}, \ldots \mathrm{x}_{n}\right)<\mathrm{y}\right] \rightarrow(\mathrm{x} \prec \mathrm{y})
$$

$(\prec, \preceq)$ :

$$
(\mathrm{x} \prec \mathrm{y}) \rightarrow(\mathrm{x} \preceq \mathrm{y})
$$

$(\leq, \preceq)$ :

$$
(\mathrm{x} \leq \mathrm{y}) \rightarrow(\mathrm{x} \preceq \mathrm{y})
$$

- Distributivity/Bijection:

$$
\begin{gathered}
\forall \mathrm{x} \in \mathrm{X}, \mathrm{y} \in \mathrm{Y} \\
{[\mathrm{G}[(\ldots \mathrm{x} \ldots)(\ldots \mathrm{y} \ldots)], \text { s.t. }} \\
\left.f_{1-1}:\left[x_{1} \rightarrow y_{1}\right], \ldots, f_{1-1}:\left[x_{n} \rightarrow y_{n}\right]\right] .
\end{gathered}
$$

10 See, e.g., Smart (1959, pp. 148-149), for an attempt to account for how phenomenal properties and biological properties can be identical, while phenomenal properties might yet have distinct higher-order properties. 
-truths about consciousness are weak part, s.t. Q $\preceq \mathrm{Q}$

- Panpsychism (in Non-constitutive guise: Phenomenal properties are the intrinsic realizers of extrinsic functional properties and their roles; in Constitutive guise: (i) fundamental microphysical entities are functionally specified and they instantiate microphenomenal properties, where microphenomenal properties are the realizers of the fundamental microphysical entity's role/functional specification; and (ii) microphenomenal properties constitute the macrophenomenal properties of macrophysical entities):

Truths about consciousness (Q) ground truths about functional role (F) if and only if the grounding operator is:

-strict full, s.t. Q $<$ F; and

-non-distributive, s.t. $\neg \exists f_{1-1}\langle\mathrm{Q}, \mathrm{F}\rangle$

The philosophical significance of the hyperintensional regimentation of the ontology of consciousness is at least three-fold. First, the regimentation permits one coherently to formulate Phenomenal Reality Type Identity. Leibniz's law states that for all propositional variables $\mathrm{x}, \mathrm{y}$ and for all properties $\mathrm{R}, \mathrm{x}=\mathrm{y}$ iff ( $\mathrm{Rx} \Longleftrightarrow \mathrm{Ry}$ ). According to the Phenomenal Realist Type Identity proposal, phenomenal properties are identicial to biological properties, while phenomenal properties are in some sense non-reductively real. Thus, in the modal setting, Phenomenal Realist Type Identity belies Leibniz's law, on the assumption that the latter can be applied to intensional entities. One virtue of the hyperintensional regimentation is thus that it avoids this result, by providing a framework with the expressive resources sufficient to formulate the non-reductive Type Identity proposal.

Second, the hyperintensional grounding regimentation evinces how functionalist approaches to the ontology of consciousness can be explanatory, because the identification of phenomenal properties with functional organization can be defined via the foregoing ground-theoretic explanatory properties. Block (2015) suggests thatby contrast to Phenomenal Realist Type Identity-identifying phenomenal properties with functional roles cannot sufficiently account for the ground-theoretic explanation of the identity. Block distinguishes between metaphysical and ontological versions of physicalism. Block's 'ontological physicalism' is a reductive, functionalist theory, and eschews of explanation by restricting the remit of its theory to 'what there is'; i.e. to specifying identity statements between entities in the domain of quantification (114). By contrast, Block's 'metaphysical physicalism' — namely, Phenomenal Realist Type Identity — purports to account for the nature of the entities figuring in theoretical identity statements via availing of relations of explanatory, ontological dependence (op. cit.).

Block poses the following consideration against the functionalist (115-117). Suppose that there is a counterpart of a human organism with isomorphic functional properties, but comprised of distinct biological properties. Suppose that the functional isomorph instantiates phenomenal properties. Block argues that the functional isomorph 'is like us superficially, but not in any deep property that can plausibly be one that scientists will one day tell us is the physical ground of consciousness [... ] So there is a key question that that kind of reductive physicalism-ontological physicalism — does not ask nor answer: what is it that creatures with the same phenom- 
enology share that grounds that phenomenology' (op. cit.)? The foregoing does not provide an argument that the neuro- and psycho-functionalist must provide an account of in virtue of what phenomenal properties are instantiated. Rather, Block suggests only that functionalist proposals do not sufficiently inquire into the realizers of the functional roles that they specify. He suggests that this theoretical approach would be insufficient, if one were to seek an explanation of the psychofunctional correlations between phenomenal property types and the relevant functional roles.

The second theoretical virtue of the hyperintensional regimentation is thus that it demonstrates how Block's analysis might be circumvented. Functionalism can be regimented within the logic of hyperintensional ground; and can therefore satisfy the formal requirements on explaining in virtue of what phenomenal truths ontologically depend upon functional truths. Cf. Khudairi (op. cit.). ${ }^{11}$

Third, and most crucially: The regimentation demonstrates how metaphysically possible relations between consciousness and physics cannot be witnessed by epistemic constraints, when the latter are recorded by the conceivability-i.e., the epistemic possibility - thereof. Propositional epistemic modality is blind to the hyperintensional, metaphysical dependencies holding between phenomenal and physical truths. Thus, the two-dimensional conceivability argument against the derivation of phenomenal truths from physical truths risks being obviated by a hyperintensional regimentation of the ontology of consciousness.

Acknowledgements For valuable comments, I am grateful to audiences at the Tucson Science of Consciousness conference, the conference on Grounding and Consciousness at the University of Birmingham, as well as to Josh Dever, David Chalmers, Kit Fine, David Papineau, and an anonymous referee.

Open Access This article is distributed under the terms of the Creative Commons Attribution 4.0 International License (http://creativecommons.org/licenses/by/4.0/), which permits unrestricted use, distribution, and reproduction in any medium, provided you give appropriate credit to the original author(s) and the source, provide a link to the Creative Commons license, and indicate if changes were made.

\section{References}

Balog, K. (1999). Conceivability, possibility, and the mind-body problem. Philosophical Review, $108,4$. Block, N. (2006). Max Black's objection to mind-body identity. In D. Zimmerman (Ed.), Oxford studies in metaphysics (Vol. 3). Oxford: Oxford University Press.

\footnotetext{
11 Of pertinence to the foregoing is another distinction drawn by Fine (2015a), between material and criterial identity conditions. While material identity conditions imply the identity of the objects in question, criterial identity conditions explain in virtue of what the objects in question are the same. In order to countenance criterial identity conditions, Fine avails of his earlier work on arbitrary objects (cf. Fine 1985, 2015b, p. 305). Let a model, $M$, for a first-order language, $L$, be a tuple, where $M=\langle I, A, R, V\rangle$, with $I$ a domain of concrete and abstract individuals, $A$ a domain of arbitrary objects, $R$ a dependence relation on arbitrary objects, and $V$ a non-empty set of partial functions from $A$ to $I$ (cf. Fine 1985). The arbitrary objects in $A$ can be conceived of as reified variables, and the dependence relation between any $a$ and $b$ in $A$ may be interpreted as a relation of ontological dependence (op. cit.: pp. 59-60). A criterial identity condition for, e.g., sets, can then be stated as follows: Given arbitrary $\mathrm{x}, \mathrm{y}$, with $\operatorname{Set}(\mathrm{x}) \wedge \operatorname{Set}(\mathrm{y}):\left[\forall \mathrm{z}(\mathrm{z} \in \mathrm{x} \equiv \mathrm{z} \in \mathrm{y}) \rightarrow_{x, y}(\mathrm{x}\right.$ $=\mathrm{y}$ )]. (Intuitively: Given arbitrary objects, $\mathrm{x}, \mathrm{y}$, whose values are sets, the fact that $\mathrm{x}$ and $\mathrm{y}$ share the same members grounds the fact that they are the same.) A crucial point of departure between the foregoing and the approach proffered in this essay is Fine's ontology of arbitrary objects, to which the present proposal need make no appeal.
} 
Block, N. (2015). The Canberra plan neglects ground. In T. Horgan, M. Sabates, \& D. Sosa (Eds.), Qualia and mental causation in a physical world: Themes from the philosophy of Jaegwon Kim. Cambridge: Cambridge University Press.

Chalmers, D. (1996). The conscious mind. Oxford: Oxford University Press.

Chalmers, D. (2010). The character of consciousness. Oxford: Oxford University Press.

Chalmers, D. (2012). Constructing the world. Oxford: Oxford Universtity Press.

Chalmers, D. (2014). Strong necessities and the mind-body problem. Philosophical Studies, 167, 3.

Chalmers, D., \& Rabern, B. (2014). Two-dimensional semantics and the nesting problem. Analysis, 74, 2.

Fine, K. (1985). Reasoning with arbitrary objects. Oxford: Blackwell Publishing.

Fine, K. (2012a). The pure logic of ground. The Review of Symbolic Logic, 5, 1.

Fine, K. (2012b). Guide to ground. In F. Correia \& B. Schnieder (Eds.), Metaphysical grounding. Cambridge: Cambridge University Press.

Fine, K. (2015a). Identity criteria and ground. Philosophical Studies. doi:10.1007/s11098-014-0440-7.

Fine, K. (2015b). Unified foundations for essence and ground. Journal of the American Philosophical Association, 1, 296-311.

Khudairi, H. (submitted). Consciousness, Haecceitism, and Grounding.

Montero, B. (2010). A Russellian response to the structural argument against physicalism. Journal of Consciousness Studies, 17, 3-4.

Rosen, G. (2010). Metaphysical dependence: Grounding and reduction. In B. Hale \& A. Hoffman (Eds.), Modality: Metaphysics, logic, and epistemology. Oxford: Oxford University Press.

Routley, R., \& Meyer, R. (1972a). The semantics of entailment II. Journal of Philosophical Logic, 1, 53-73.

Routley, R., \& Meyer, R. (1972b). The semantics of entailment III. Journal of Philosophical Logic, 1, 192-208.

Routley, R., \& Meyer, R. (1973). The semantics of entailment I. In H. Leblanc (Ed.), Truth, syntax, and semantics. Amsterdam: North-Holland.

Seager, W. (1995). Consciousness, information, and panpsychism. Journal of Consciousness Studies, 2, $272-288$.

Skiles, A. (2015). Against grounding necessitarianism. Erkenntnis, 80, 717-751.

Smart, J. J. C. (1959). Sensations and brain processes. Philosophical Review, 68, 2.

Stoljar, D. (2001). Two conceptions of the physical. Philosophy and Phenomenological Research, 62, 2.

Stoljar, D. (2014). Four kinds of Russellian monism. In U. Kriegel (Ed.), Contemporary debates in philosophy of mind. London: Routledge.

Strawson, G. (2006). Realistic monism. Journal of Consciousness Studies, 13, 3. 